\title{
Qualitative behavior of a variable-yield simple food chain with an inhibiting nutrient
}

\author{
S. R.-J. Jang ${ }^{1}$, J. Baglama ${ }^{2}$ \\ Department of Mathematics and Statistics, Texas Tech University, Lubbock, \\ Texas 79409-1042 U.S.A.
}

\begin{abstract}
A simple food chain which consists of nutrient, prey and predator in which nutrient is growth limiting at low concentrations but growth inhibiting at high concentrations is investigated in this study. It is assumed that the nutrient concentration is separated into internal and external nutrient concentation and only the internal nutrient level is capable of catalyzing cell growth. It is shown that the dynamics of the system depend on thresholds $R_{0}$ and $R_{1}$. With inhibition, there exist initial conditions for which the predator becomes extinct but not the prey when $R_{0}<1$. If $R_{0}, R_{1}>1$, the system is uniformly persistent even in the inhibited environment.
\end{abstract}

Keywords: cell-quota; inhibition; threshold; uniform persistence

\section{Introduction}

Several nutrients such as nitrate, nitrite and ammonia limit growth of microorganisms at low concentrations and are inhibitory or even toxic at high concentrations $[1,2,3,4]$. This inhibitory mechanism of substrates has a profound impact on the living organism. It is the purpose of this work to investigate the inhibition upon the dynamics of a nutrient-prey-predator model.

In most ecological models, it is assumed that the consumption rate of a resource is a function of resource availability and the yield of the organism is constantly proportional to the amount consumed. Such a model is sometimes referred to as a constant-yield model. However, it has been observed in both laboratory and field studies that algal populations could survive for several weeks after external reserves of nutrients were depleted. This is a result of luxury consumption of nutrients, first described by Ketchum [5]. The ability 
of algae to store nutrient in excess of their requirements indicates that the growth rate of algae may not respond at once to a change in the external concentration of nutrients.

The Droop model, also called the variable-yield model, effectively decouples specific growth rate of the organism from external nutrient concentration by introducing an intracellular store of nutrient, providing a good fit for microorganism growth [6]. However, unlike the classical constantyield models, the global dynamics of the variable-yield models were not well understood until recently. Lange and Oyarzum $[7,8]$ provided a global analysis for a variable-yield chemostat model with a single species of microorganism. Smith and Waltman [9, 10] studied two species of microorganism competing for a single limiting nutrient in a chemostat, where they showed that the competitive exclusion principal remains valid. Smith [11] also studied a periodically forced Droop model with a single species of organism in a chemostat. Jang [12] examined several variable-yield nutrientphytoplankton-zooplankton models.

Certain nutrients are growth limiting at low concentrations but growth inhibiting or even toxic at high concentrations; nitrate is one example. Kumar et al. [3] include an additional factor $\exp \left(-c \mathrm{NO}_{3}\right)$ in the uptake rate to denote this inhibition by nitrate. Other substrates such as nitrite and ammonia are also known to be inhibitory to some bacteria at high concentrations [2]. In addition, industrial waste products such as phenols and thiocyanates are often inhibitory to the bacteria metabolizing them $[1,13]$. Several researchers use a nonmonotone uptake rate to model this inhibition.

Chemostat systems with nonmonotone uptake rates consisting of two trophic levels, a substrate and $n$ competing species, have been studied by Butler and Wolkowicz [14] and Wolkowicz and Lui [15]. Jang and Allen [16] examined a simple food chain of three trophic levels with nonmonotone uptake rate for the prey. Other studies include Aris and Humphrey [17], Boon and Laudelout [18] and Bush and Cook [13]. In these studies, they assumed that the yield of the microorganism is constantly proportional to the external nutrient consumed, i.e., they considered constant-yield models.

Our simple food chain studied here consists of a nutrient, prey and predator, where prey feeds on the nutrient and predator feeds on the prey. Moreover, we separate nutrient concentrations into external and internal nutrient level and only the internal nutrient can catalyze cell growth. That is, we use the Droop equation for prey to study the interplay between nutrient, prey and predator. In this model, both prey and predator are modelled in terms of 
their nutrient content and it is assumed that there is no net nutrient loss due to death or due to nutrient conversion. Under these assumptions, the total nutrient content is constant for all time and so the system is closed. Furthermore, it is also assumed that the nutrient limits growth at low concentrations but inhibits growth at high concentrations. We use a nonmonotone uptake rate for the prey to model this inhibition.

It has been shown in [12] that if there is no inhibition, the dynamics of the model are very similar to that of classical epidemic models [19]. Particularly, there exist two thresholds $R_{0}$ and $R_{1}$ such that the system is uniformly persistent if $R_{0}$ and $R_{1}>1$. Our focus in this study will be on the inhibitory mechanism. In particular, it is shown that if $R_{0}<1$, there exists a threshold initial prey density for the nutrient-prey model for which the prey can survive in a steady state fashion if the initial population density is above the threshold. On the other hand, if the initial prey population is too small, the population becomes extinct. Thus the inhibition of nutrient has an inverse density dependence effect on the prey population. It requires the prey population to detoxify its environment by consuming enough nutrient so as to lower nutrient concentration. Therefore, if its initial population is not sufficiently large, the prey has no ability to detoxify its habitat and consequently the population becomes extinct. The same conclusion is also valid for the nutrient-prey-predator model. If $R_{0}>1$, we can define one more threshold $R_{1}$. If $R_{1}>1$, then the variable-yield nutrient-prey-predator system is uniformly persistent even in the inhibited environment.

In the following section, a variable-yield nutrient-prey model is derived and analyzed. Section 3 studies a variable-yield simple food chain. Specifically, we use a Lotka-Volterra functional response as the predator's uptake rate. Section 4 provides a numerical example and computer simulations. The conclusion is given in the final section.

\section{$2 \quad$ A nutrient-prey model}

In this section, we adopt internal nutrient storage by prey to study the dynamics of a nutrient-prey model with inhibition. We use a nonmonotone uptake function to model nutrient inhibition. The model derivation here is parallel to that of monotone uptake in [12].

Unlike the classical constant-yield model, the specific growth rate of the

prey depends on a quantity, $Q(t)$, called the "cell quota", introduced by 
Droop $[20,21]$, which can be viewed as the average amount of stored nutrient in each cell of the prey at time $t$. The uptake rate of prey is assumed to depend on both the external and the internal nutrient concentrations.

Let $N(t)$ be the free nutrient at time $t$ and $P(t)$, the concentration (or number of cells) of prey at time $t$. Then $P(t) Q(t)$ is the total amount of stored nutrient at time $t$. Let $u(Q)$ and $\rho(N, Q)$ be the per-capita growth rate and the per-capita uptake rate of prey respectively. Motivated by the examples of functions $u$ and $\rho$ in the literature $[9,10,20,21,22,23,24,25,26,27]$ and taking into account of inhibition, we make the following assumptions:

(H1) there exists $Q_{0}>0$ such that $u\left(Q_{0}\right)=0, u^{\prime}(Q)>0$ and $u^{\prime}$ is continuous for $Q \geq Q_{0}$.

(H2) $\rho \in C^{1}(N, Q)$ for $N \geq 0, Q \geq Q_{0} ; \rho(0, Q)=0$ for $Q \geq Q_{0}$; $\rho(N, Q) \geq 0$ and $\frac{\partial \rho}{\partial Q} \leq 0$ for $N \geq 0, Q \geq Q_{0}$. There exists $N_{0}>0$ such that $\frac{\partial \rho}{\partial N}>0$ for $0 \leq N<N_{0}, Q \geq Q_{0}$ and $\frac{\partial \rho}{\partial N}<0$ for $N>N_{0}, Q \geq Q_{0}$.

We shall refer to the nutrient concentration $N_{0}$ defined in $(\mathrm{H} 2)$ as the toxic level. It is the level at which the nutrient becomes growth inhibiting.

Let $\delta$ be the death rate of prey. Since the prey is modeled in terms of nutrient level and we assume that there is no nutrient loss to the system due to death, the interplay between nutrient and prey is described by the following ordinary differential equations:

$$
\begin{aligned}
\dot{N} & =-P \rho(N, Q)+\delta P Q \\
\dot{P} & =P[u(Q)-\delta] .
\end{aligned}
$$

Let $\sum(t)$ be the total amount of free and stored nutrient at time $t$, i.e., $\sum(t)=N(t)+P(t) Q(t)$. Since it is assumed that the system is closed, the total nutrient is thus constant, i.e., $\dot{\sum}(t)=0$ for $t \geq 0$. This implies that $P \dot{Q}=P[\rho(N, Q)-u(Q) Q]$ and consequently $\dot{Q}=\rho(N, Q)-u(Q) Q$ if $P(t) \neq 0$ for all $t \geq 0$. Therefore, we have derived a variable-yield nutrientprey model

$$
\begin{aligned}
\dot{N} & =-P \rho(N, Q)+\delta P Q \\
\dot{P} & =P[u(Q)-\delta] \\
\dot{Q} & =\rho(N, Q)-u(Q) Q .
\end{aligned}
$$


As the total nutrient is constant, i.e., $\sum(t)=N_{T}$ for all $t \geq 0$, system (2.2) is equivalent to the following two-dimensional system

$$
\begin{aligned}
\dot{P} & =P[u(Q)-\delta] \\
\dot{Q} & =\rho\left(N_{T}-P Q, Q\right)-u(Q) Q .
\end{aligned}
$$

Equation for $\dot{P}$ in $(2.1)$ implies that $P(t)=P(0) \exp \left(\int_{0}^{t}[u(Q(\tau))-\delta] d \tau\right)$ and thus $P(t)>0$ for all $t \geq 0$ if $P(0)>0$. Note that when $P=0$, there is no biologically meaningful equation for $Q$. However, the equation for $Q$ makes mathematical sense even if $P=0$ as $P=0$ and $Q=\hat{Q}$ where $\hat{Q}$ satisfies $\rho\left(N_{T}, Q\right)=u(Q) Q$, resulting in a biological equilibrium.

Let $F=\left\{(P, Q) \in R_{+}^{2}: P Q \leq N_{T}, Q \geq Q_{0}\right\}$. It is straightforward to show that $F$ is positively invariant for (2.3) and that system (2.3) is dissipative. Moreover, steady state $E_{0}=(0, \hat{Q})$ always exists with $\hat{Q}$ defined as above. Suppose $u(Q)=\delta$ has a solution $Q_{1}$ and consider

$$
\rho\left(N_{T}-P Q_{1}, Q_{1}\right)=\delta Q_{1} .
$$

If $N_{T} \leq N_{0}$, then the left hand side of (2.4) is strictly decreasing and thus (2.3) has a positive steady state $E_{11}=\left(P_{1}, Q_{1}\right)$ if and only if $\rho\left(N_{T}, Q_{1}\right)>$ $\delta Q_{1}$. In which case, the positive steady state is unique. If $N_{T}>N_{0}$, observe that the left hand side of (2.4) is strictly decreasing if $P>\frac{N_{T}-N_{0}}{Q_{1}}$ and is strictly increasing if $0 \leq P<\frac{N_{T}-N_{0}}{Q_{1}}$. Thus if $\delta Q_{1}<\rho\left(N_{T}, Q_{1}\right)$, then (2.3) has a unique positive steady state $E_{11}=\left(P_{1}, Q_{1}\right)$, where $N_{T}-P_{1} Q_{1}<N_{0}$. However, if $\rho\left(N_{T}, Q_{1}\right)<\delta Q_{1}<\rho\left(N_{0}, Q_{1}\right)$, then system (2.3) has two positive steady states $E_{11}=\left(P_{1}, Q_{1}\right)$ and $E_{12}=\left(P_{2}, Q_{1}\right)$, where $N_{T}-P_{2} Q_{1}>N_{0}$. On the other hand, if $\delta Q_{1}>\rho\left(N_{0}, Q_{1}\right)$, then system (2.3) has no positive steady state.

In order to discuss the global dynamics of (2.3), the following observation is useful.

Proposition 2.1 There exists $Q_{1}>Q_{0}$ such that $u\left(Q_{1}\right)=\delta$ and $\rho\left(N_{T}, Q_{1}\right)>$ $\delta Q_{1}$ if and only if $u(\hat{Q})>\delta$.

Proof. To show necessity, suppose $u(\hat{Q}) \leq \delta$. Then $\hat{Q} \leq Q_{1}$ and thus $\rho\left(N_{T}, \hat{Q}\right) \geq \rho\left(N_{T}, Q_{1}\right)>\delta Q_{1} \geq u(\hat{Q}) \hat{Q}$, a contradiction. Hence $u(\hat{Q})>\delta$. Conversely, if $u(\hat{Q})>\delta$, then $u(Q)=\delta$ has a solution $Q_{1}$. Furthermore, $\rho\left(N_{T}, Q_{1}\right) \geq \rho\left(N_{T}, \hat{Q}\right)=u(\hat{Q}) \hat{Q}>\delta \hat{Q}>\delta Q_{1}$. 
A simple calculation shows that the Jacobian matrix of (2.3) is given by

$$
\left(\begin{array}{cc}
u(Q)-\delta & P u^{\prime}(Q) \\
-Q \partial \rho / \partial N & -P \partial \rho / \partial N+\partial \rho / \partial Q-u(Q)-u^{\prime}(Q) Q
\end{array}\right)
$$

where $\partial \rho / \partial N$ and $\partial \rho / \partial Q$ are evaluated at $\left(N_{T}-P Q, Q\right)$.

Let $Q^{*}$ satisfy $\rho\left(N_{0}, Q\right)=u(Q) Q$. Note that $Q^{*}$ is always well-defined. If $u(\hat{Q})<\delta$, then it follows from Proposition 2.1 that $\rho\left(N_{T}, Q_{1}\right)<\delta Q_{1}$ if $Q_{1}$ exists. Therefore if $u(\hat{Q})<\delta$ and $Q_{1}$ exists, we have either $\rho\left(N_{T}, Q_{1}\right)<$ $\rho\left(N_{0}, Q_{1}\right)<\delta Q_{1}$ or $\rho\left(N_{T}, Q_{1}\right)<\delta Q_{1}<\rho\left(N_{0}, Q_{1}\right)$. The global dynamics of (2.3) is given in the following theorem.

Theorem 2.2 The dynamics of (2.3) can be summarized as the following.

(1) Let $N_{T} \leq N_{0}$. In addition if

(a) $u(\hat{Q})<\delta$, then $E_{0}$ is the only steady state and every solution of (2.3) converges to $E_{0}$.

(b) $u(\hat{Q})>\delta$, then $E_{0}, E_{11}$ both exist and every solution of (2.3) with $P(0)>0$ converges to $E_{11}$.

(2) Let $N_{T}>N_{0}$ and $u(\hat{Q})>\delta$. Then steady states $E_{0}, E_{11}$ both exist and every solution of (2.3) with $P(0)>0$ converges to $E_{11}$.

(3) Let $N_{T}>N_{0}$ and $u(\hat{Q})<\delta$.

(a) If $Q_{1}$ does not exist, then $E_{0}$ is the only steady state and every solution converges to $E_{0}$.

(b) If $Q_{1}$ exists and $\rho\left(N_{T}, Q_{1}\right)<\rho\left(N_{0}, Q_{1}\right)<\delta Q_{1}$, then $E_{0}$ is the only steady state and every solution converges to $E_{0}$.

(c) If $Q_{1}$ exists and $\rho\left(N_{T}, Q_{1}\right)<\delta Q_{1}<\rho\left(N_{0}, Q_{1}\right)$, then there are two positive steady states $E_{1 i}=\left(P_{i}, Q_{1}\right), 1 \leq i \leq 2$. The stable manifold of $E_{12}$ separates $F$ into two disjoint subsets $F_{1}$ and $F_{2}$ such that $E_{0}$ is a global attractor in $F_{1}$ and $E_{11}$ is a global attractor in $F_{2}$.

Proof. (1) This case has been previously studied in [9, 10]. Since (2.3) is a dissipative planar system, the proof is straightforward. Note that $E_{11}$ exists if and only if $u(\hat{Q})>\delta$ by Proposition 2.1 and as $\partial \dot{P} / \partial P+\partial \dot{Q} / \partial Q=$ 
$-\delta-P \partial \rho / \partial N+\partial \rho / \partial Q-u^{\prime}(Q) Q<0$ for $(P, Q) \in F$, it follows from the Dulac criterion that there is no periodic solution in $F$ if $u(\hat{Q})>\delta$. This completes the proof of (1)

(2) If $N_{T}>N_{0}$ and $u(\hat{Q})>\delta$, then $\rho\left(N_{T}, Q_{1}\right)>\delta Q_{1}$ by Proposition 2.1 and thus there exists a unique positive steady state $E_{11}=\left(P_{1}, Q_{1}\right)$. Define a subset $\Gamma$ of $F$ by $\Gamma=\left\{(P, Q) \in F: Q<Q^{*}, N_{T}-P Q<N_{0}\right\}$. Observe that $Q_{1}<\hat{Q}<Q^{*}$ and $E_{11} \in \Gamma$.

Furthermore, as $\left.\dot{Q}\right|_{Q=Q^{*}}=\rho\left(N_{T}-P Q^{*}, Q^{*}\right)-u\left(Q^{*}\right) Q^{*} \leq \rho\left(N_{0}, Q^{*}\right)-$ $u\left(Q^{*}\right) Q^{*}=0$ for all $P \geq 0$ and $\left.(P Q)\right|_{P Q=N_{T}-N_{0}, Q<Q^{*}}=P\left[-\delta Q+\rho\left(N_{T}-\right.\right.$ $P Q, Q)]\left.\right|_{P Q=N_{T}-N_{0}, Q<Q^{*}}=\left.P\left[\rho\left(N_{0}, Q\right)-\delta Q\right]\right|_{P Q=N_{T}-N_{0}, Q<Q^{*}}>P\left[\rho\left(N_{0}, Q^{*}\right)-\right.$ $\left.\delta Q^{*}\right]\left.\right|_{P Q=N_{T}-N_{0}, Q<Q^{*}}=\left.P\left[u\left(Q^{*}\right) Q^{*}-\delta Q^{*}\right]\right|_{P Q=N_{T}-N_{0}, Q<Q^{*}}>0$, solutions of (2.3) with $P(0)>0$ enter $\Gamma$ in finite time and remain there for all future time. Consequently, as $\partial \dot{P} / \partial P+\partial \dot{Q} / \partial Q<0$ for $(P, Q) \in \Gamma$, the Dulac criterion implies that there is no periodic solution in $\Gamma$. We conclude that $E_{11}$ is a global attractor in $F \backslash Q$-axis.

(3) (a) If $Q_{1}$ does not exist, then $E_{0}$ is the only steady state. Since (2.3) is dissipative, it follows from the Poincaré-Bendixson Theorem that $E_{0}$ is a global attractor.

(b) In this case $\rho\left(N_{T}-P Q_{1}, Q_{1}\right) \leq \rho\left(N_{0}, Q_{1}\right)<\delta Q_{1}$ for all $P \geq 0$, there is no positive steady state and thus $E_{0}$ is a global attractor for (2.3).

(c) Notice that both positive steady states $E_{11}=\left(P_{1}, Q_{1}\right)$ and $E_{12}=$ $\left(P_{2}, Q_{1}\right)$ exist. Also $E_{0}$ and $E_{11}$ are local attractors and $E_{12}$ is a saddle point. The stable manifold of $E_{12}$ separates $F$ into two disjoint subsets $F_{1}$ and $F_{2}$ such that $E_{0} \in F_{1}$ and $E_{11} \in F_{2}$. Since there is no positive steady state in $F_{1}, E_{0}$ is a global attractor in $F_{1}$. In the following we show that $E_{11}$ is a global attractor in $F_{2}$.

Let $Q^{*}$ be defined as above. Clearly, $Q^{*}>\hat{Q}$. If $Q^{*} \leq Q_{1}$, then $\rho\left(N_{T}-P_{1} Q_{1}, Q_{1}\right)=u\left(Q_{1}\right) Q_{1} \geq u\left(Q^{*}\right) Q^{*}=\rho\left(N_{0}, Q^{*}\right) \geq \rho\left(N_{0}, Q_{1}\right)>$ $\rho\left(N_{T}-P_{1} Q_{1}, Q_{1}\right)$, a contradiction. Thus $\hat{Q}<Q_{1}<Q^{*}$. Let $\Gamma_{1}=\{(P, Q) \in$ $\left.F_{2}: Q<Q^{*}, P Q>N_{T}-N_{0}\right\}$. Then $E_{11} \in \Gamma_{1}$. Observe that $\left.\dot{Q}\right|_{Q=Q^{*}}=$ $\rho\left(N_{T}-P Q^{*}, Q^{*}\right)-u\left(Q^{*}\right) Q^{*} \leq 0$ for all $P \geq 0$ and $\left.(\dot{P} Q)\right|_{P Q=N_{T}-N_{0}, Q<Q^{*}}=$ $\left.P\left[\rho\left(N_{0}, Q\right)-\delta Q\right]\right|_{P Q=N_{T}-N_{0}, Q<Q^{*}}>\left.P\left[\rho\left(N_{0}, Q^{*}\right)-\delta Q^{*}\right]\right|_{P Q=N_{T}-N_{0}, Q<Q^{*}}>0$. Any solution of (2.3) with initial condition in $F_{2}$ enters $\Gamma_{1}$ in finite time and remains there for all future time. Furthermore, the Dulac criterion can be applied to show that there is no periodic solution in $\Gamma_{1}$. Therefore, any solution of $(2.3)$ with initial condition in $F_{2}$ converges to $E_{11}$. 
We now define a threshold $R_{0}=\frac{u(\hat{Q})}{\delta}$. If $R_{0}>1$, then with or without inhibition, the prey population can survive. If $R_{0}<1$ and without inhibition, the prey population becomes extinct. However, with inhibition and $R_{0}<1$, it was shown in $(3)(\mathrm{c})$ of Theorem 2.2 that there exist initial populations for which the prey can stabilize in a positive equilibrium fashion.

The result of Theorem $2.2(3)(\mathrm{c})$ is very interesting. In addition to the stable boundary equilibrium $E_{0}=(0, \hat{Q})$, there are two interior equilibria $E_{1 i}=\left(P_{i}, Q_{1}\right), i=1,2$. Equilibrium $E_{11}$ is always locally asymptotically stable with $N_{T}-P_{1} Q_{1}<N_{0}$ and $\frac{\partial \rho}{\partial N}\left(N_{T}-P_{1} Q_{1}, Q_{1}\right)>0$. Therefore, the nutrient stimulates uptake at $E_{11}$. On the other hand, equilibrium $E_{12}$ is always unstable with $N_{T}-P_{2} Q_{1}>N_{0}$ and $\frac{\partial \rho}{\partial N}\left(N_{T}-P_{2} Q_{1}, Q_{1}\right)<0$. Thus the nutrient inhibits uptake at $E_{12}$. The stable manifold of $E_{12}$ (as is illustrated in Figure 2) becomes the threshold initial density for population persistence. The prey can survive in a steady state fashion if the initial population density is above the threshold. Otherwise, the prey population is too small to detoxify its environment and consequently becomes extinct. The inhibition of nutrient thus has an inverse density dependent effect on the prey population and the persistence threshold is no longer a single number. This complicated threshold behavior is due to the physiological adjustments of cell quota and uptake rate which are made in the variable-yield model.

In the following, we illustrate this particular mechanism. Figure 1 provides the graphs of $\rho(N, Q)$ as a function of $N$ for different values of $Q$ when $Q=Q_{0}, Q=\hat{Q}, Q=Q_{1}$ and $Q=Q^{*}$. The vertical lines $N=N_{0}, N=N_{T}$ and the horizontal line $y=\delta Q_{1}$ are also drawn to identify three possible equilibria $E_{0}, E_{11}$ and $E_{12}$. At $E_{12}$, the physiological adjustment for equilibrium occurs in an inhibited inverse density dependent population, so that $E_{12}$ is unstable. At $E_{11}$, the physiological adjustment takes place in a density dependent population, which makes $E_{11}$ stable. Therefore, if the initial population is too small to detoxify its habitat, the population approaches $E_{12}$, but is unable to complete its adjustment and is thus tends toward $E_{0}=(0, \hat{Q})$. As $u(\hat{Q})<\delta$, the prey population is doomed to extinction. 


\section{A nutrient-prey-predator model}

In this section we study the dynamics of a variable-yield nutrient-preypredator model with inhibition. We assume that the predator's uptake rate is modelled by a Lotka-Volterra functional response. Let $b$ be the maximal predator ingestion rate, $d$ the predator net nutrient conversion rate and $\epsilon$ the predator death rate. Clearly, $d \leq b$. The model derivation here is parallel to that of the monotone case in [12]. We assume that the ecosystem is closed. In particular, the prey which is removed through predator's predation becomes either a part of the available external nutrient or predator's biomass. The interplay between external nutrient, prey and predator can be described by the following ordinary differential equations

$$
\begin{aligned}
\dot{N} & =-P \rho(N, Q)+\delta P Q+\epsilon Z+(b-d) P Q Z \\
\dot{P} & =P[u(Q)-\delta]-b P Z \\
\dot{Z} & =Z(d P Q-\epsilon) .
\end{aligned}
$$

As the system is closed, i.e., $P(t) Q(t)+Z(t)+N(t)=N_{T}$ for all $t \geq 0$, we can derive an equation for the cell quota $\dot{Q}=\rho(N, Q)-u(Q) Q$ as we did in section 2. Consequently, the four dimensional system is equivalent to the following three dimensional system

$$
\begin{aligned}
\dot{P} & =P[u(Q)-\delta-b Z] \\
\dot{Q} & =\rho\left(N_{T}-P Q-Z, Q\right)-u(Q) Q \\
\dot{Z} & =Z(d P Q-\epsilon) .
\end{aligned}
$$

In addition to (H2), we make the following technical assumption:

(H3) $\rho\left(N_{T}-\epsilon / d, Q_{0}\right)<\delta Q_{0}$ if $N_{T}>\epsilon / d$.

Let $\Omega=\left\{(P, Q, Z) \in R_{+}^{3}: Q \geq Q_{0}, P Q+Z \leq N_{T}\right\}$ and $Q^{*}$ satisfy $\rho\left(N_{0}, Q\right)=u(Q) Q$. Then $\Omega$ is positively invariant for (3.2) and in fact $\hat{\Omega}=\left\{(P, Q, Z) \in \Omega: P \leq \frac{N_{T}}{Q_{0}}, Z \leq N_{T}, Q \leq Q^{*}\right\}$ is a compact global attractor for system (3.2) if $N_{T} \geq N_{0}$ and $K=\{(P, Q, Z) \in \Omega: P \leq$ $\left.\frac{N_{T}}{Q_{0}}, Z \leq N_{T}, Q \leq \hat{Q}\right\}$ is a compact global attractor for $(3.2)$ if $N_{T}<N_{0}$.

As predator has no effect on the boundary steady states, the existence conditions for steady states $E_{0}=(0, \hat{Q}, 0), E_{11}=\left(P_{1}, Q_{1}, 0\right)$ and $E_{12}=$ 
$\left(P_{2}, Q_{1}, 0\right)$ are the same as previous section. For the existence of a positive steady state $(\bar{P}, \bar{Q}, \bar{Z})$, it is necessary that $N_{T}>\epsilon / d$, where $\bar{Q}$ must satisfy

$$
\rho\left(N_{T}-\epsilon / d-\frac{u(Q)-\delta}{b}, Q\right)=u(Q) Q .
$$

Note that (3.3) is always solvable for $Q$. But since $\rho(N, Q)$ is not monotone in $N, \bar{Q}$ may not be unique. Moreover, a positive steady state $E_{2}=(\bar{P}, \bar{Q}, \bar{Z})$ exists if and only if $u(\bar{Q})>\delta$. In which case $\bar{P}=\frac{\epsilon}{d \bar{Q}}$ and $\bar{Z}=\frac{u(\bar{Q})-\delta}{b}$.

The Jacobian matrix of (3.2) has the following form

$$
\left(\begin{array}{ccc}
u(Q)-\delta-b Z & P u^{\prime}(Q) & -b P \\
-Q \partial \rho / \partial N & -P \partial \rho / \partial N+\partial \rho / \partial Q-u(Q)-u^{\prime}(Q) Q & -\partial \rho / \partial N \\
d Q Z & d P Z & d P Q-\epsilon
\end{array}\right),
$$

where $\partial \rho / \partial N$ and $\partial \rho / \partial Q$ are evaluated at $\left(N_{T}-P Q-Z, Q\right)$. In particular, the Jacobian matrix at $E_{0}$ is given by

$$
\left(\begin{array}{ccc}
u(\hat{Q})-\delta & 0 & 0 \\
-\hat{Q} \partial \rho / \partial N & \partial \rho / \partial Q-u(\hat{Q})-u^{\prime}(\hat{Q}) \hat{Q} & -\partial \rho / \partial N \\
0 & 0 & -\epsilon
\end{array}\right)
$$

where $\partial \rho / \partial N$ and $\partial \rho / \partial Q$ are evaluated at $\left(N_{T}, \hat{Q}\right)$ and the Jacobian matrix at $E_{1 i}$ is given by

$$
\left(\begin{array}{ccc}
0 & P_{i} u^{\prime}\left(Q_{1}\right) & -b P_{i} \\
-Q_{1} \partial \rho / \partial N & -P_{i} \partial \rho / \partial N+\partial \rho / \partial Q-u\left(Q_{1}\right)-u^{\prime}\left(Q_{1}\right) Q_{1} & -\partial \rho / \partial N \\
0 & 0 & d P_{i} Q_{1}-\epsilon
\end{array}\right)
$$

where $\partial \rho / \partial N$ and $\partial \rho / \partial Q$ are evaluated at $\left(N_{T}-P_{i} Q_{1}, Q_{1}\right)$ for $1 \leq i \leq 2$. Consequently, it is easy to see that $E_{0}$ is a local attractor if $u(\hat{Q})<\delta$. If $u(\hat{Q})>\delta$, then $E_{0}$ is a saddle point with stable manifold lying in the $Q-Z$ plane. $E_{11}$ is a local attractor if $P_{1} Q_{1}<\epsilon / d$. If $P_{1} Q_{1}>\epsilon / d$, then $E_{11}$ is a saddle point which is unstable in the positive direction orthogonal to the $P$-Q-plane. Moreover, $E_{12}$ is always a saddle point. The stable manifold of $E_{12}$ is two dimensional if $P_{2} Q_{1}<\epsilon / d$ and is one dimensional if $P_{2} Q_{1}>\epsilon / d$.

System (3.2) is said to be persistent if $\liminf _{t \rightarrow \infty} P(t)>0, \liminf _{t \rightarrow \infty} Q(t)$ $>Q_{0}$ and $\liminf _{t \rightarrow \infty} Z(t)>0$ for any solution $(P(t), Q(t), Z(t))$ of $(3.2)$ with $P(0)>0, Q(0)>Q_{0}$ and $Z(0)>0$. (3.2) is said to be uniformly persistent if 
there exists $\hat{a}>0$ such that for any solution of $(3.2)$ with $P(0)>0, Q(0)>$ $Q_{0}$ and $Z(0)>0$ we have $\liminf _{t \rightarrow \infty} P(t) \geq \hat{a}, \liminf _{t \rightarrow \infty} Q(t) \geq \hat{a}$ and $\liminf _{t \rightarrow \infty} Z(t) \geq \hat{a}$.

If $N_{T} \leq N_{0}$, then since $\partial \rho / \partial N>0$ for $0 \leq N<N_{T}, Q \geq Q_{0}$, there is no inhibition effect. The dynamics of (3.2) is given by the following theorem which is cited from [12, Theorem 2.3, 2.4 and 2.5].

Theorem 3.1 If $N_{T} \leq N_{0}$, then the dynamics of (3.2) can be summarized as the following.

(1) If $u(\hat{Q})<\delta$, then $E_{0}=(0, \hat{Q}, 0)$ is the only steady state and it is a global attractor for (3.2).

(2) If $u(\hat{Q})>\delta$, then $E_{0}=(0, \hat{Q}, 0)$ and $E_{11}=\left(P_{1}, Q_{1}, 0\right)$ both exist. If in addition $P_{1} Q_{1}<\epsilon / d$, then there is no positive steady state and every solution of (3.2) with $P(0)>0$ converges to $E_{11}$. If $P_{1} Q_{1}>\epsilon / d$, then there exists a unique positive steady state which is a local attractor. Moreover, system (3.2) is uniformly persistent.

If $N_{T}>N_{0}$, we separate our discussion into $u(\hat{Q})>\delta$ and $u(\hat{Q})<\delta$. We first discuss the case when $u(\hat{Q})>\delta$.

Theorem 3.2 If $N_{T}>N_{0}$ and $u(\hat{Q})>\delta$, then $E_{0}=(0, \hat{Q}, 0)$ and $E_{11}=$ $\left(P_{1}, Q_{1}, 0\right)$ both exist.

(1) If $P_{1} Q_{1}<\epsilon / d$, then there is no positive steady state and every solution of (3.2) with $P(0)>0$ converges to $E_{11}$.

(2) If $P_{1} Q_{1}>\epsilon / d$, then system (3.2) is uniformly persistent.

Proof. It follows from Proposition 2.1 that $Q_{1}$ exists and $\rho\left(N_{T}, Q_{1}\right)>\delta Q_{1}$. Thus $E_{11}=\left(P_{1}, Q_{1}, 0\right)$ is the only steady state on the positive $P$ - $Q$ plane, where $N_{T}-P_{1} Q_{1}<N_{0}$. Moreover, since $u(\hat{Q})>\delta, E_{0}$ is a saddle point with stable manifold lying in the positive $Q-Z$ plane.

(1) If $P_{1} Q_{1}<\epsilon / d$, then $E_{11}$ is a local attractor. We first show that there is no positive steady state. Clearly if $N_{T} \leq \epsilon / d$, then there is no positive steady state. We assume $N_{T}>\epsilon / d$ and let $\bar{Q}$ satisfy (3.3). If $\bar{Q}>Q_{1}$, then since $N_{T}-P_{1} Q_{1}<N_{0}$ and $P_{1} Q_{1}<\epsilon / d, N_{T}-\epsilon / d<N_{0}$. Consequently, $u(\bar{Q}) \bar{Q}=\rho\left(N_{T}-\epsilon / d-\frac{u(\bar{Q})-\delta}{b}, \bar{Q}\right) \leq \rho\left(N_{T}-P_{1} Q_{1}, \bar{Q}\right) \leq$ 
$\rho\left(N_{T}-P_{1} Q_{1}, Q_{1}\right)=u\left(Q_{1}\right) Q_{1}$, a contradiction. We conclude that there is no positive steady state. On the other hand, since the positive $P-Q$ plane is positively invariant, by using an argument as in the proof of Theorem 2.2, it can be shown that $E_{11}$ is a global attractor in the positive $P-Q$ plane. It remains to show that solutions of $(3.2)$ with $P(0)>0$ converge to $E_{11}$.

Observe that if $N_{T} \leq \epsilon / d$, then $\dot{Z}(t) \leq 0$ for $t \geq 0$ and thus $\lim _{t \rightarrow \infty} Z(t)=$ $z^{*} \geq 0$. If $z^{*}>0$, then since $\lim _{t \rightarrow \infty} \dot{Z}(t)=0, \lim _{t \rightarrow \infty} P(t) Q(t)=\epsilon / d$, an immediate contradiction. Thus $\lim _{t \rightarrow \infty} Z(t)=0$ and (1) is shown. Otherwise, let $\tilde{\Omega}=\{(P, Q, Z) \in \Omega: P>0\}$ and partition $\tilde{\Omega}$ into three pairwise disjoint subsets: $\tilde{\Omega}_{1}=\{(P, Q, Z) \in \tilde{\Omega}: P Q<\epsilon / d\}, \tilde{\Omega}_{2}=\{(P, Q, Z) \in \tilde{\Omega}$ : $P Q=\epsilon / d\}$ and $\tilde{\Omega}_{3}=\{(P, Q, Z) \in \tilde{\Omega}: P Q>\epsilon / d\}$. If there exists a trajectory which remains in $\tilde{\Omega}_{3}$ for all large time, then $Z(t) \geq 0$ for all $t$ large and thus $\lim _{t \rightarrow \infty} Z(t)=z^{*} \geq 0$. Since $\ddot{Z}(t)$ is bounded above, $\lim _{t \rightarrow \infty} \dot{Z}(t)=0$. Thus if $z^{*}>0$, then $\lim _{t \rightarrow \infty} P(t) Q(t)=\epsilon / d$, i.e., the $\omega$-limit set of such a trajectory lies in the set $C=\left\{\left(P, Q, z^{*}\right) \in \tilde{\Omega}: P Q=\epsilon / d\right\}$. We need to show that the only nonempty invariant, connected subset in $C$ is a point.

Indeed, let $\tilde{C}$ be an arbitrary invariant, connected subset of $C$ such that $\left(\hat{p}, \hat{q}, z^{*}\right)$ and $\left(\tilde{p}, \tilde{q}, z^{*}\right) \in \tilde{C}$. We may assume $\hat{p}<\tilde{p}$ and thus $\hat{q}>\tilde{q}$. Hence any $p, q$ with $\hat{p}<p<\tilde{p}, \hat{q}>q>\tilde{q}$ and $p q=\epsilon / d,\left(p, q, z^{*}\right)$ is also in $\tilde{C}$ by connectedness. However, $0=\left.(\dot{P} Q)\right|_{P Q=\epsilon / d, Z=z^{*}}=P\left[-\delta Q-b Q Z+\rho\left(N_{T}-\right.\right.$ $P Q-Z, Q)]\left.\right|_{P Q=\epsilon / d, Z=z^{*}}$ if and only if $\rho\left(N_{T}-\epsilon / d-z^{*}, Q\right)=\delta Q+b Q z^{*}$. This latter equation has at most one solution. We conclude that $\tilde{C}$ consists of at most one point. Since the $\omega$-limit set is nonempty and there is no positive steady state, $z^{*}=0$. But then such a trajectory would converge to $E_{11}$. We thus obtain a contradiction and conclude that no trajectory remains in $\tilde{\Omega}_{3}$ for all large time.

Suppose a trajectory crosses $\tilde{\Omega}_{2}$ at time $t_{0}$. Since $N_{T}-\epsilon / d<N_{T}-$ $P_{1} Q_{1}<N_{0}, \quad(\dot{P Q})\left(t_{0}\right)=\left[P \rho\left(N_{T}-P Q-Z, Q\right)-\delta P Q-b P Q Z\right]\left(t_{0}\right) \leq$ $P\left[\rho\left(N_{T}-P Q-Z, Q\right)-\delta Q\right]\left(t_{0}\right)=P\left[\rho\left(N_{T}-\epsilon / d-Z, Q\right)-\delta Q\right]\left(t_{0}\right) \leq P\left[\rho\left(N_{T}-\right.\right.$ $\epsilon / d, Q)-\delta Q]\left(t_{0}\right)<0$ for all $Q \geq Q_{0}$ by $(\mathrm{H} 3)$, such a trajectory enters $\tilde{\Omega}_{1}$ and remains there for all future time. Consequently, $\dot{Z}(t) \leq 0$ for all $t$ large and $\lim _{t \rightarrow \infty} Z(t)=z^{*} \geq 0$. Similar argument as in the previous paragraph can be applied to show that $z^{*}=0$ and hence $E_{11}$ is a global attractor in $\tilde{\Omega}$.

(2) To show uniform persistence of system (3.2), we apply criterion derived by Thieme [28]. Let $X_{1}=\left\{(P, Q, Z) \in \Omega: P>0, Q>Q_{0}, Z>0\right\}$ and $X_{2}=\Omega \backslash X_{1}$. Then $X_{1}$ is open and positively invariant for (3.2). By using the Jacobian matrix at $E_{11}$ and the fact that $P_{1} Q_{1}>\epsilon / d, E_{11}$ is unstable 
in the positive direction orthogonal to the $P-Q$ plane. A result of Freedman and Waltman [29] shows that system (3.2) is persistent, i.e., $X_{2}$ is a strong repeller for $X_{1}$ and hence a weak repeller for $X_{1}$. Since (3.2) has a compact global attractor $\hat{\Omega}$, applying Theorem 4.4 of Thieme [28] and conclude that $X_{2}$ is a uniform weak repeller for $X_{1}$. Proposition 1.2 of Thieme [28] can then be used to show that $X_{2}$ is a uniform strong repeller for $X_{1}$. Therefore, we conclude that system (3.2) is uniformly persistent.

We next consider the case when $N_{T}>N_{0}$ and $u(\hat{Q})<\delta$. Proposition 2.1 implies that $\rho\left(N_{T}, Q_{1}\right)<\delta Q_{1}$ if $Q_{1}$ exists. Therefore if $Q_{1}$ exists, then either $\rho\left(N_{T}, Q_{1}\right)<\rho\left(N_{0}, Q_{1}\right)<\delta Q_{1}$ or $\rho\left(N_{T}, Q_{1}\right)<\delta Q_{1}<\rho\left(N_{0}, Q_{1}\right)$ can occur.

Theorem 3.3 If $N_{T}>N_{0}$ and $u(\hat{Q})<\delta$. The dynamics of (3.2) can be summarized as the following.

(1) If $Q_{1}$ does not exist, then $E_{0}$ is the only steady state and it is a global attractor.

(2) If $Q_{1}$ exists and such that $\rho\left(N_{T}, Q_{1}\right)<\rho\left(N_{0}, Q_{1}\right)<\delta Q_{1}$, then $E_{0}$ is the only steady state and it is a global attractor.

(3) If $Q_{1}$ exists and such that $\rho\left(N_{T}, Q_{1}\right)<\delta Q_{1}<\rho\left(N_{0}, Q_{1}\right)$, then $E_{11}=$ $\left(P_{1}, Q_{1}, 0\right)$ and $E_{12}=\left(P_{2}, Q_{1}, 0\right)$ both exist. If in addition $P_{1} Q_{1}<\epsilon / d$, then except a set of initial conditions of Lebesgue measure 0 , solutions of (3.2) either converge to $E_{0}$ or to $E_{11}$.

Proof. Since $u(\hat{Q})<\delta, E_{0}$ is a local attractor.

(1) In this case $u(Q)<\delta$ for all $Q \geq Q_{0}$ and thus $\dot{P}(t) \leq 0$ for all $t \geq 0$. Hence $\lim _{t \rightarrow \infty} P(t)=p^{*} \geq 0$ exists. Since $\ddot{P}(t)$ is bounded below, $\lim _{t \rightarrow \infty} \dot{P}(t)=0$. Hence if $p^{*}>0$, then it follows from system (3.2) and the assumption of (1) that $\lim _{t \rightarrow \infty} Q(t)=\infty$. We arrive at a contradiction. Thus $\lim _{t \rightarrow \infty} P(t)=0$ and hence $\lim _{t \rightarrow \infty} Z(t)=0$, i.e., $E_{0}$ is a global attractor for $(3.2)$.

(2) If $\rho\left(N_{T}, Q_{1}\right)<\rho\left(N_{0}, Q_{1}\right)<\delta Q_{1}$, then $\rho\left(N_{T}-P Q_{1}, Q_{1}\right) \leq \rho\left(N_{0}, Q_{1}\right)<$ $\delta Q_{1}$ and there is no steady state of the form $(P, Q, 0)$. Let $Q^{*}$ satisfy $\rho\left(N_{0}, Q\right)=u(Q) Q$. If $Q^{*} \geq Q_{1}$, then $\rho\left(N_{0}, Q^{*}\right)=u\left(Q^{*}\right) Q^{*} \geq u\left(Q_{1}\right) Q_{1}=$ $\delta Q_{1}>\rho\left(N_{0}, Q_{1}\right) \geq \rho\left(N_{0}, Q^{*}\right)$, a contradiction. Hence if $N_{T}>\epsilon / d$ and $\bar{Q}$ satisfies (3.3), then since $\rho\left(N_{T}-\epsilon / d-\frac{u(Q)-\delta}{b}, Q\right) \leq \rho\left(N_{0}, Q\right)$ for all $Q \geq Q_{0}$, 
$\bar{Q} \leq Q^{*}<Q_{1}$, i.e., $u(\bar{Q})<\delta$. We conclude that (3.2) has no positive steady state.

To show that $E_{0}$ is a global attractor for (3.2), we partition $\Omega$ into three pairwise disjoint subsets: $\Omega_{1}=\left\{(P, Q, Z) \in \Omega: Q<Q_{1}\right\}, \Omega_{2}=\{(P, Q, Z) \in$ $\left.\Omega: Q=Q_{1}\right\}$ and $\Omega_{3}=\left\{(P, Q, Z) \in \Omega: Q>Q_{1}\right\}$. If there exists a trajectory which remains in $\Omega_{3}$ for all large time, then $\dot{Q}=\rho\left(N_{T}-P Q-Z, Q\right)-u(Q) Q<$ $\rho\left(N_{T}-P Q-Z, Q_{1}\right)-u\left(Q_{1}\right) Q_{1} \leq \rho\left(N_{0}, Q_{1}\right)-\delta Q_{1}<0$ for all large time. Hence $\lim _{t \rightarrow \infty} Q(t)=q^{*} \geq Q_{1}$ exists. Since $\ddot{Q}(t)$ is bounded below, $\lim _{t \rightarrow \infty} \dot{Q}(t)=$ 0 . But as $\dot{Q}(t)<\rho\left(N_{0}, Q_{1}\right)-\delta Q_{1}<0$ we obtain a contradiction. Therefore, no trajectory remains in $\Omega_{3}$ for all large time. Suppose there exists $t_{0} \geq 0$ such that $Q\left(t_{0}\right)=Q_{1}$. Then $\dot{Q}\left(t_{0}\right)<\rho\left(N_{0}, Q_{1}\right)-\delta Q_{1}<0$. Such a trajectory enters $\Omega_{1}$ and remains there for all future time. A similar argument as in (1) can then be applied to show that $E_{0}$ is a global attractor.

(3) There are two steady states of the form $E_{11}=\left(P_{1}, Q_{1}, 0\right)$ and $E_{12}=$ $\left(P_{2}, Q_{1}, 0\right)$. If $P_{1} Q_{1}<\epsilon / d$, then $E_{11}$ is a local attractor and $E_{12}$ is a saddle point with two dimensional stable manifold. Similar analysis as in the proof of Theorem 3.2 can be used to show that there is no positive steady state. To show our desired result, it is enough to show that $\lim _{t \rightarrow \infty} Z(t)=0$ for all solution of (3.2). The idea of the proof is similar to that (1) of Theorem 3.2.

Clearly if $N_{T} \leq \epsilon / d$, then $\lim _{t \rightarrow \infty} Z(t)=0$. We now assume $N_{T}>\epsilon / d$. We partition $\Omega$ into three pairwise disjoint subsets: $\Delta_{1}=\{(P, Q, Z) \in \Omega$ : $P Q<\epsilon / d\}, \Delta_{2}=\{(P, Q, Z) \in \Omega: P Q=\epsilon / d\}$ and $\Delta_{3}=\{(P, Q, Z) \in$ $\Omega: P Q>\epsilon / d\}$. If there exists a trajectory which remains in $\Delta_{3}$ for all large time, then $\dot{Z}(t) \geq 0$ for all large time and thus $\lim _{t \rightarrow \infty} Z(t)=z^{*} \geq 0$ exists. If $z^{*}>0$, then $\lim _{t \rightarrow \infty} P(t) Q(t)=\epsilon / d$, i.e., the $\omega$-limit set of this trajectory lies in $C=\left\{\left(P, Q, z^{*}\right) \in \Omega: P Q=\epsilon / d\right\}$. We can show that the only nonempty invariant, connected subset in $C$ is a point. Since there is no positive steady state, $z^{*}=0$. But then we obtain a contradiction. Similarly, if there exists $t_{0}>0$ such that $P\left(t_{0}\right) Q\left(t_{0}\right)=\epsilon / d$, then it can be shown that $(\dot{P Q})\left(t_{0}\right)<0$. We conclude that every trajectory of $(3.2)$ will enter $\Delta_{1}$ and remain there for all large time. Hence, $\lim _{t \rightarrow \infty} Z(t)=0$ is shown. Note that restricted on the boundary where $Z=0,(3.2)$ becomes (2.3). Therefore, except a set of initial conditions of Lebesgue measure 0, every trajectory of (3.4) either converges to $E_{0}$ or to $E_{11}$ by Theorem $2.2(3)(\mathrm{c})$.

Note that Theorem 3.3 does not discuss the cases for which $P_{2} Q_{1}<$ $\epsilon / d<P_{1} Q_{1}$ or $\epsilon / d<P_{2} Q_{1}$. As $u(\hat{Q})<\delta, E_{0}$ is a local attractor. We can immediately conclude that system (3.2) is not persistent. Numerical example 
in the next section revails that the system is indeed not persistent for either of these cases.

As in section 2 , we let $R_{0}=\frac{u(\hat{Q})}{\delta}$. If $N_{T} \leq N_{0}$ and $R_{0}<1$, the prey population becomes extinct as was shown in Theorem 3.1. For $N_{T}>N_{0}$ and $R_{0}<1$, if either $u(Q)<\delta$ for $Q \geq Q_{0}$ or $Q_{1}$ exists and $\rho\left(N_{0}, Q_{1}\right)<\delta Q_{1}$, then the prey population become extinct and so does the predator. Otherwise, there are two steady states $E_{11}=\left(P_{1}, Q_{1}, 0\right)$ and $E_{12}=\left(P_{2}, Q_{1}, 0\right)$ and we can define two more thresholds $R_{1 i}=\frac{d P_{i} Q_{1}}{\epsilon}, 1 \leq i \leq 2$. It is known that $R_{12}<R_{11}$. If $R_{11}<1$, then predator can not survive and there are initial conditions for which the prey population can stabilize in an equlibrium fashion. That is, the inhibition allows the prey to survive at a concentration level which is too low for predator survival. In the next section, numerical simulations will demonstrate that if either $R_{12}<1<R_{11}$ or $R_{12}>1$, the system is not persistent.

If $R_{0}>1$, since there is only one steady state of the form $(P, Q, 0)$, we define threshold $R_{1}=\frac{d P_{1} Q_{1}}{\epsilon}$. If $R_{1}<1$, then only the prey population can survive. However, if $R_{1}>1$, both prey and predator can exist in coexistence with each other in either the inhibited or the uninhibited environment.

\section{Numerical Simulations}

A very preliminary numerical study of model (3.2) is given in [30]. In this section we provide a more detailed numerical simulation. In fact, we use numerical examples to illustrate analytical results of Theorem 2.2, Theorem 3.2 and Theorem 3.3.

We adopt growth rate $u$ and uptake rate $\rho$ taken from Grover [26, 27] and incorporated with inhibition

$$
\begin{gathered}
u(Q)=u_{\max } \frac{\left(Q-Q_{\min }\right)_{+}}{k+\left(Q-Q_{\min }\right)_{+}} \\
\rho(N, Q)=\rho_{\max }(Q) \frac{N}{N^{2}+N+4},
\end{gathered}
$$


where $\rho_{\max }(Q)=\rho_{\max }^{\text {high }}-\left(\rho_{\max }^{\text {high }}-\rho_{\max }^{\text {low }}\right) \frac{\left(Q-Q_{\min }\right)_{+}}{Q_{\max }^{-Q} \min }$ and $\left(Q-Q_{\min }\right)_{+}$ denotes the positive part of $Q-Q_{\min }$, i.e., $\left(Q-Q_{\min }\right)_{+}=Q-Q_{\min }$ if $Q \geq Q_{\min }$ and $\left(Q-Q_{\min }\right)_{+}=0$ if $Q<Q_{\min }$. Specific parameter values are $\rho_{\max }^{\text {high }}=15, \rho_{\max }^{\text {low }}=0.9, k=2, Q_{\min }=3, Q_{\max }=30$ and $u_{\max }=2.16$. From (4.2), it is known that the toxic level $N_{0}$ is 2 . The nutrient-prey model is then given by

$$
\begin{aligned}
\dot{P} & =P\left[2.16 \frac{(Q-3)_{+}}{2+(Q-3)_{+}}-\delta\right] \\
\dot{Q} & =\left[15-0.522(Q-3)_{+}\right] \frac{N_{T}-P Q}{\left(N_{T}-P Q\right)^{2}+\left(N_{T}-P Q\right)+4}-2.16 \frac{(Q-3)_{+} Q}{2+(Q-3)_{+}}
\end{aligned}
$$

We use parameter values $\delta=0.35$ and $N_{T}=15$. Then $\hat{Q}=3.294274$ and $u(\hat{Q})=0.2771<\delta=0.35$. Moreover, $Q_{1}=3.3867$ exists with $\rho\left(N_{T}, Q_{1}\right)=$ $0.9097<\delta Q_{1}=1.1854<\rho\left(N_{0}, Q_{1}\right)=2.9596$. Thus $E_{0}=(0, \hat{Q}), E_{11}=$ $\left(P_{1}, Q_{1}\right)$ and $E_{12}=\left(P_{2}, Q_{1}\right)$ all exist with $P_{1}=4.3229$ and $P_{2}=1.1443$. Fig.2 shows that the stable manifold of $E_{12}$ separates $\tilde{F}=\left\{(P, Q) \in R_{+}^{2}\right.$ : $P Q \leq 15\}$ into disjoint subsets $\tilde{F}_{1}$ and $\tilde{F}_{2}$ such that solutions with initial conditions in $\tilde{F}_{1}$ converge to $E_{0}$ and solutions with initial conditions in $\tilde{F}_{2}$ converge to $E_{11}$. Therefore the separatrix shown in Figure 2 becomes the threshold for population persistence.

For the nutrient-prey-predator model, the system is given below

$$
\begin{aligned}
\dot{P} & =P\left[2.16 \frac{(Q-3)_{+}}{2+(Q-3)_{+}}-\delta-b Z\right] \\
\dot{Q} & =\left[15-0.522(Q-3)_{+}\right] \frac{N_{T}-P Q-Z}{\left(N_{T}-P Q-Z\right)^{2}+\left(N_{T}-P Q-Z\right)+4}-2.16 \frac{(Q-3)_{+} Q}{2+(Q-3)_{+}} \\
\dot{Z} & =Z(d P Q-\epsilon) .
\end{aligned}
$$

We use $N_{T}=3.5, \delta=0.2$ and $b=0.8$. In this case, $Q_{1}=3.2041, \hat{Q}=$ $3.8858, u(\hat{Q})=0.663078>\delta=0.2$ and $P_{1}=1.0358$. If we let $\epsilon=0.35$ and $d=0.1$, then $P_{1} Q_{1}=3.3188<\frac{\epsilon}{d}=3.5$. Fig.3(a) shows that solutions with $P(0)>0$ converge to $E_{11}=\left(P_{1}, Q_{1}, 0\right)$. If we choose $\epsilon=0.35$ and $d=0.8$, then $P_{1} Q_{1}>\frac{\epsilon}{d}=0.4375$. Fig.3(b) shows that solutions with positive initial 
conditions are eventually trapped in a compact subset which is a positive distance from the boundary.

We next use $N_{T}=10$. If $\delta=0.8$, then $Q_{1}=4.1765, \hat{Q}=3.4249$ and $u(\hat{Q})=0.3785<\delta=0.8$. Moreover, $\rho\left(N_{T}, Q_{1}\right)=1.3002<\rho\left(N_{0}, Q_{1}\right)=$ $2.8771<\delta Q_{1}=3.3412$. Fig.4(a) demonstrates that solutions converge to $E_{0}=(0, \hat{Q}, 0)$. If we choose $\delta=0.4$, then $Q_{1}=3.4545$ and $\rho\left(N_{T}, Q_{1}\right)=$ $1.2950<\delta Q_{1}=1.3818<\rho\left(N_{0}, Q_{1}\right)=2.9525$. Thus $E_{11}=\left(P_{1}, Q_{1}, 0\right)$ and $E_{12}=\left(P_{2}, Q_{1}, 0\right)$ both exist with $P_{1}=2.7696$ and $P_{2}=0.2167$. If $\epsilon=0.97$ and $d=0.1$, then $P_{1} Q_{1}=9.5676<\frac{\epsilon}{d}=9.7$. Fig.4(b) illustrates that solutions either converge to $E_{0}$ or to $E_{11}$.

Finally, we let $N_{T}=10, \delta=0.4$ and $b=0.1$. If $\epsilon=0.41$ and $d=0.05$, then $P_{2} Q_{1}=0.7486<\frac{\epsilon}{d}=8.2<P_{1} Q_{1}=9.5676$. There exists a unique positive steady state $E_{2}=(2.2608,3.6270,1.155)$ and there are trajectories which converge to $E_{2}$. However, system (4.4) is not persistent. For example, the solution with $P(0)=0.6, Q(0)=2.1$ and $Z(0)=8$ converges to $E_{0}$ as is shown in Fig.5(a). Similarly, if we choose $\epsilon=0.05$ and $d=0.1$, then $P_{1} Q_{1}>P_{2} Q_{1}>\frac{\epsilon}{d}=0.5$ and system (4.4) is not persistent, as is shown in Fig.5(b). This is in part due to the fact that $u(\hat{Q})<\delta$ and $E_{0}$ is a local attractor.

\section{Discussion}

A variable-yield simple food chain with a growth inhibiting nutrient is derived and analyzed. The model provides us a better understanding of the impact of inhibition upon the dynamics of a simple nutrient-prey-predator system with internal nutrient storage by prey.

Our analytical results can be interpreted in terms of several thresholds. If $R_{0}<1$, the system in general is not persistent, i.e., there exist solutions with $P(0)>0, Z(0)>0$ such that either $P(t) \rightarrow 0$ as $t \rightarrow \infty$ and consequently $Z(t) \rightarrow 0$ as $t \rightarrow \infty$ or only $Z(t) \rightarrow 0$ as $t \rightarrow \infty$. In certain cases as was shown in (3) of Theorem 3.3 we can define two more thresholds given by $R_{1 i}=\frac{d P_{i} Q_{1}}{\epsilon}$. Each $R_{1 i}$ can be viewed as the basic reproductive number of predator when prey's population is stabilized at $P_{i}$. If $R_{11}<1$, then there exist initial populations for which the predator becomes extinct but not the prey under the inhibited environment. This inhibition provides prey 
an environment to survive at a density level which is too low for predator survival. If either $R_{12}<1<R_{11}$ or $1<R_{12}<R_{11}$, then system (3.2) is not persistent. This is a direct implication of local stability of steady state $E_{0}$. However, computer simulation demonstrates that there do exist initial populations with large prey densities for which both prey and predator become extinct.

If $R_{0}>1$, we can define one more threshold $R_{1}=\frac{d P_{1} Q_{1}}{\epsilon}$. If $R_{1}<1$, only the prey population can survive. If $R_{1}>1$, the system is uniformly persistent. However, the dynamics are much more complicated than simply converging to a steady state as illustrated in Fig.3(b) of section 4.

Although a simple Lotka-Volterra functional response is used as the predator's uptake rate, our study of a variable-yield simple food chain with nonmonotone uptake functions provided here gives us an insight of how inhibition alters the dynamics of a simple food chain without inhibition. The results obtained here can be applied to nutrient-based population models for which inhibition is likely. In particular, they are applicable to toxicology and bioremediation where microbial food chain and consortia are used to detoxify contaminants.

Acknowledgements

We are grateful to the referees for their valuable comments and suggestions for the biological interpretations of Theorem 2.2 (3)(c). 


\section{Footnote}

1. Corresponding author. Tel.: +1-806 742-1422; e-mail: rjang@math.ttu.edu

2. Current address: Department of Mathematical Sciences, Ball State University, Muncie, IN 47306; e-mail: jbaglama@math.bsu.edu 


\section{References}

[1] J.F. Andrews, A mathematical model for the continuous culture of microorganisms utilizing inhibitory substrates, Biotech. Bioengrg. 10 (1968) 707-723.

[2] J.L. Jost, J.F.Drake, A.G. Fredrickson, H.M. Tsuchiya, Interactions of Tetrahymena pyriformis, Escherichia coli, Azotobacter vinelandii, and glucose in a minimal medium, J. Bacteriol. 113 (1973) 834-840.

[3] S.K. Kumar, W.F. Vincent, P.C. Austin, G.C. Wake, Picoplankton and marine food chain dynamics in a variable mixed-layer: a reactiondiffusion model, Ecol. Modelling 57 (1991) 193-219.

[4] D.R. Yang, A.E. Humphrey, Dynamic and steady-state studies of phenol biodegradation in pure and mixed cultures, Biotech. Bioengrg. 17 (1975) 1211-1235.

[5] B.H. Ketchum, The absorption of phosphate and nitrate by illuminated cultures of Nitzschia Clsterium, Amer. J. Botany 26 (1939) 399-407.

[6] D.L. DeAngelis, Dynamics of Nutrient Cycling and Food Webs, Chapman \& Hall, New York, 1992.

[7] K. Lange, F.J. Oyarzum, The attractiveness of the Droop equations, Math. Biosci. 111 (1992) 261-278.

[8] F.J. Oyarzum, K. Lange, The attractiveness of the Droop equations II. Generic uptake and growth functions, Math. Biosci. 121 (1994) 127-139.

[9] H.L. Smith, P. Waltman, Competition for a single limiting resource in continuous culture: The variable-yield model, SIAM J. Appl. Math. 54 (1994) 1113-1131.

[10] H.L. Smith, P. Waltman, The Theory of the Chemostat, Cambridge University Press, New York, 1995.

[11] H.L. Smith, The periodically forced Droop model for phytoplankton growth in a chemostat, J. Math. Biol. 35 (1997) 545-556. 
[12] S. Jang, Dynamics of variable-yield nutrient-phytoplankton-zooplankton models with nutrient recycling and self-shading, J. Math. Biol. (to appear).

[13] A. W. Bush, A.E. Cook, The effect of time delay and growth rate inhibition in the bacterial treatment of wastewater, J. Theor. Biol. 63 (1976) 385-395.

[14] G.J. Butler, G.S.K. Wolkowicz, A mathematical model of the chemostat with a general class of functions describing nutrient uptake, SIAM J. Appl. Math. 45 (1985) 138-151.

[15] G.S.K. Wolkowicz, Z. Lu, Global dynamics of a mathematical model of competition in the chemostat: General response functions and differential death rates, SIAM J. Appl. Math. 52 (1992) 222-233.

[16] S. Jang, L. Allen, A simple food chain with a growth inhibiting nutrient, Appl. Math. Comput. 104 (1999) 277-298.

[17] R. Aris, A.E. Humphrey, Dynamics of a chemostat in which two organisms compete for a common substrate, Biotech. Bioengrg. 19 (1977) 1375-1386.

[18] B. Boon, H. Laudelout, Kinetics of nitrite oxidation by Nitrobacter winogradskyi, Biochem J. 85 (1962) 440-447.

[19] H.W. Hethcote, Qualitative analysis of communicable disease models, Math. Biosci. 28 (1976) 335-356.

[20] M.R. Droop, Some thoughts on nutrient limitation in algae, J. Phycology 9 (1973) 264-272.

[21] M.R. Droop, The nutrient status of algae cells in continuous culture, J. Marine Biol. Asso. 54 (1974) 825-855.

[22] J.W. Caperon, J. Meyer, Nitrogen-limited growth of marine phytoplankton I. Changes in population characteristics with steady-state growth rate, Deep-Sea Res. 19 (1972) 601-618.

[23] J.W. Caperon, J. Meyer, Nitrogen-limited growth of marine phytoplankton II. Uptake kinetics and their role in nutrient-limited growth of phytoplankton, Deep-Sea Res. 19 (1972) 619-632. 
[24] A. Cunningham, R.M. Nisbet, Time lag and co-operativity in the transient growth dynamics of microalgae, J. Theoret. Biol. 84 (1983) 189-203.

[25] A. Cunningham, R.M. Nisbet, Transients and oscillations in continuous cultures, in: M.J. Bazin, (Ed.), Mathematical Microbiology, Academic Press, New York, 1983, pp.77-103.

[26] J.P. Grover, Resource competition in a variable environment- phytoplankton growing according to the variable-internal-stores model, Amer. Nat. 138 (1991) 811-835.

[27] J.P. Grover, Constant- and variable-yield models of population growthResponses to environmental variability and implications for competition, J. Theoret. Biol. 158 (1992) 409-428.

[28] H.R. Thieme, Persistence under relaxed point-dissipativity (with application to an endemic model), SIAM J. Math. Anal. 24 (1993) 407-435.

[29] H.I. Freedman, P. Waltman, Persistence in models of three interacting predator-prey populations, Math. Biosci. 68 (1984) 213-231.

[30] S. Jang, A mathematical model and computer simulations of the interactions between microbes, Proceedings 11th IEEE symposium on computer based medical systems (1998) 151-155. 


\section{Figure legends}

Figure1: Graphs of functions $\rho(N, Q)$ for $Q=Q_{0}, Q=\hat{Q}, Q=Q_{1}$ and $Q=Q^{*}$ are plotted against $N$. Three possible equilibria $E_{0}, E_{11}$ and $E_{12}$ are shown as occurs in Theorem $2.2(3)(\mathrm{c})$, where $E_{0}$ and $E_{11}$ are stable and $E_{12}$ is unstable.

Figure2: Plot of trajectories of system (4.3) using $\delta=0.35$ and $N_{T}=$ 15. Solutions of (4.3) either converge to $E_{0}=(0,3.294274)$ or to $E_{11}=$ $(4.3229,3.3867)$ with the exception of the stable manifold of $E_{12}=(1.1443,3.3867)$.

Figure3: Trajectories of system (4.4) plotted using $N_{T}=3.5, b=0.8$ and $\delta=0.2$. In part (a), $\epsilon=0.35$ and $d=0.1$. Solutions of (4.4) with positive initial conditions converge to $E_{11}=(1.0358,3.2041,0)$. In part (b), $\epsilon=0.35$ and $d=0.8$. Solutions of (4.4) seem to converge to a periodic solution.

Figure4: Plot of trajectories of system (4.4) using $N_{T}=10$. In part (a) $\delta=0.8$ and all solutions converge to $E_{0}=(0,3.4249,0)$. In part (b), $\delta=0.4$. Solutions either converge to $E_{0}=(0,3.4249,0)$ or to $E_{11}=$ $(2.7696,3.4545,0)$.

Figure5: Trajectories of system (4.4) plotted using $N_{T}=3.5, b=0.8$ and $\delta=0.2$. In part (a), $\epsilon=0.35$ and $d=0.1$. Solutions of (4.4) with positive initial conditions converge to $E_{11}=(1.0358,3.2041,0)$. In part (b), $\epsilon=0.35$ and $d=0.8$. Solutions of (4.4) seem to converge to a periodic solution. 
(a) 
(b) 
(a)

28 
(b) 
(a) 
(b) 